\title{
CAMBIOS EN LAS CARACTERÍSTICAS FUnCIONALES DE EPÍFITAS VASCULARES DE BOSQUE MESÓFILO DE MONTAÑA Y VEGETACIÓN SECUNDARIa EN LA REGIÓN CENTRAL de VeracruZ, MÉXICO
}

\author{
Tania M. Susan-Tepetlan, Nó́ VelázQuez-Rosas ${ }^{1}$ y Thorsten Krömer \\ Centro de Investigaciones Tropicales, Universidad Veracruzana, Xalapa, Veracruz, México \\ ${ }^{1}$ Autor para la correspondencia: novelazquez@uv.mx
}

\begin{abstract}
Resumen: En los bosques mesófilos de montaña, los disturbios antropogénicos afectan la abundancia y diversidad de las comunidades de epífitas; sin embargo, las causas de estos cambios han sido poco exploradas. En este trabajo se evalúa la variación del área foliar, grosor de la lámina foliar, masa seca por unidad de área, densidad foliar y contenido de humedad en las diez especies de epífitas vasculares más abundantes de bosque mesófilo y vegetación derivada (bosque conservado, acahual de 20 años y cafetal bajo sombra), ubicado en la región central de Veracruz, México. El propósito del estudio fue entender de qué manera la variación mircroambiental (temperatura, humedad relativa y radiación fotosintéticamente activa), determinada por la apertura del dosel que provocan las actividades antrópicas, influye en las características funcionales de las epífitas. Las condiciones de mayor estrés hídrico y mayor radiación se registraron en la comunidad más modificada y con mayor apertura del dosel (cafetal), y disminuyeron conforme se incrementa el grado de conservación y el dosel es más cerrado (acahual-bosque). Estos cambios microambientales se asociaron con las respuestas funcionales de las especies estudiadas. En el bosque conservado, las epífitas presentaron significativamente hojas más delgadas, con mayor contenido de humedad, menor masa seca y densidad foliar; mientras que en el cafetal se encontró el patrón opuesto. Las características funcionales de las comunidades de epífitas fueron útiles para determinar el estado de conservación en el bosque mesófilo y, permitieron entender que factores microambientales determinan sus respuestas funcionales.
\end{abstract}

Palabras clave: bromelias, gradiente de conservación, helechos, orquídeas, rasgos morfo-ecofisiológicos.

\begin{abstract}
The abundance and diversity of epiphytic communities in the tropical montane cloud forests are commonly affected by anthropogenic disturbances. However, the exact causes triggering such changes in the communities have poorly been explored. Canopy structure changes is one of the main factors affecting the communities of epiphytes, but plant responses to micro-environmental factors are largely unknown. In a landscape dominated by anthropogenic changes, we aimed to understand the effects of microclimatic factors (temperature, relative humidity and photosynthetically active radiation) on the functional responses (variation in leaf area, leaf blade thickness, dry mass per unit area, leaf density and moisture content) of the ten most abundant species of epiphytes. This study was done in Central Veracruz, Mexico, and included a well-conserved cloud forest, a 20-year-old secondary forest and a shade coffee plantation. Hydric stress and radiation were correlated with canopy openness, being the coffee plantation the most stressful community for the epiphytes and the conserved forest the less stressful site. We detected plant specific functional responses to microclimatic factors, but in general, in the natural forest epiphytes presented thinner leaves with higher moisture content, lower dry mass and density, whereas in the coffee plantation the opposite pattern was found. Besides, understanding the functional responses of the species to the micro-environmental factors, the functional traits of the epiphytic communities were useful surrogates of the conservation status of the sites.
\end{abstract}

Key words: bromeliads, conservation gradient, ferns, morpho-ecophysiological traits, orchids.

a gran abundancia y diversidad de plantas epífitas, incluyendo orquídeas, aráceas, bromelias, peperomias y helechos, entre otras, es una de las características más relevantes de los bosques húmedos tropicales (Benzing, 1990;
Krömer et al., 2005). Se estima que representan el 9\% de la flora vascular y, en algunos casos, pueden llegar a formar el $50 \%$ de especies en estos bosques (Kelly et al., 1994; Zotz, 2013). Además, son muy importantes por su papel en los 
ciclos de agua y nutrientes en el dosel (Coxson y Nadkarni, 1995; Köhler et al., 2007; Hietz, 2010), así como por la amplia gama de interacciones que mantienen con la fauna (Benzing, 1990). En los bosques mesófilos de montaña, en México, son uno de los grupos mejor representados, ya que cerca del 32\% de sus plantas vasculares son epífitas (Rzedowski, 1996). Para el centro de Veracruz se reportan al menos 604 especies de epífitas (Flores-Palacios et al., 2011).

En la actualidad, la deforestación de los bosques tropicales es una de las principales causas de la pérdida de biodiversidad (MEA, 2005). En el caso de las epífitas, existe un amplio número de estudios que describen el impacto negativo en su diversidad y distribución, ocasionado por la destrucción y fragmentación de su hábitat (Turner et al., 1994; Hietz, 1998; Gradstein, 2008; Köster et al., 2009). En general, la riqueza se ve disminuida a mayor perturbación antrópica, mientras que la composición y abundancia relativa varían ampliamente entre sitios (Barthlott et al., 2001; Krömer y Gradstein, 2003; Werner et al., 2005; Wolf, 2005; Hietz et al., 2006; Flores-Palacios y García-Franco, 2008). Sin embargo, existen pocos estudios que analicen las respuestas funcionales (morfo-ecofisiológicas) de las epífitas a los cambios ambientales, causados por la deforestación o los cambios en la estructura del dosel, que afectan su supervivencia y determinan cambios en su distribución y abundancia (Hietz, 1998; Foster, 2001; Kessler, 2001; Zotz y Bader, 2009). El análisis de las características funcionales es relevante porque ayuda a entender, de manera integral, las respuestas de las plantas a distintas presiones selectivas ( $p$. ej. factores físicos o bióticos) y sus efectos en los procesos ecosistémicos (Díaz et al., 2006). En el caso de las epífitas, este enfoque de estudio puede ser relevante porque son muy sensibles a pequeñas variaciones a nivel microclimático (Pittendrigh, 1948; Benzing, 1998; Cardelús y Chazdon, 2005), por lo que se han considerado como especies indicadoras de la calidad del hábitat (Turner et al., 1994; Nadkarni y Solano, 2002; Krömer et al., en prensa). Por ejemplo, se ha observado que las especies con características xeromórficas son más abundantes en sitios con mayor estrés hídrico, por lo que la distribución de estas especies en estratos verticales es consecuencia de sus características funcionales (Andrade y Nobel, 1997; Krömer et al., 2007; Larrea y Werner, 2010; Toledo-Aceves et al., 2012). También se ha documentado que muchas especies de epífitas muestran importantes cambios anatómicos y fisiológicos cuando existe una reducción de nutrientes o cambios en la intensidad lumínica (Benzing, 2000; Hietz y Wanek, 2003; Hietz y Briones, 2004).

La masa seca por unidad de área es un rasgo funcional clave en el crecimiento de las plantas, debido a que está relacionada con las estrategias de conservación de nutrientes, agua y captación de luz (Poorter et al., 2009). En muchas especies, el incremento de la masa seca por unidad de área está relacionado a condiciones de estrés hídrico, alta radiación o condiciones oligotróficas (Niinemets et al., 1998; Niinemets et al., 2004, Poorter et al., 2009). Por ello, algunos autores han sugerido que este carácter puede ser utilizado como un indicador de las respuestas de las plantas a la variación ambiental y el estado de conservación de las comunidades vegetales del trópico húmedo (Garnier et al., 2004; Niinemets et al., 2004; Martínez-Garza et al., 2005). Para entender las causas ambientales que determinan los cambios en la masa seca es importante analizar sus componentes principales ( $p$. ej. densidad y grosor de la lámina foliar), con el propósito de evaluar las modificaciones en la estructura anatómica de las hojas que son determinadas por los factores ambientales (Poorter et al., 2009). En helechos epífitos se ha registrado un incremento en el grosor de la fronda cuando se encuentran en las zonas más expuestas del dosel (Hietz y Briones, 1998), en otras especies se han registrado incrementos en las capas celulares del clorénquima y epidermis, así como paredes celulares más gruesas en sitios con mayor estrés hídrico (Zotz y Andrade, 2002).

En México, el estado de Veracruz es el tercero con mayor superficie de bosques mesófilos de montaña (CONABIO, 2010), y este bosque es el ecosistema con mayor número de especies por unidad de área (Rzedowski, 1996). La zona central de Veracruz es la que resguarda el área más amplia de este tipo de vegetación; sin embargo, las comunidades primarias de estos bosques representan menos del 10\% de la superficie total, mientras que las zonas de vegetación secundaria y los cafetales tradicionales son las más comunes (Cruz et al., 2010). En esta región, la presión antrópica sobre estos bosques es muy fuerte: las amenazas más importantes son la ganadería, la tala selectiva, la producción industrial de café y la extracción de productos no maderables como las epífitas (Flores-Palacios y Valencia-Díaz, 2007; WilliamsLinera, 2007; Cruz et al., 2010). Debido a la gran variación que existe en la conservación de las comunidades de bosque mesófilo de esta región, es relevante realizar estudios del impacto de las actividades antrópicas sobre las epífitas. En particular, entender qué factores ambientales afectan las respuestas funcionales de las epífitas, ayudaría a establecer estrategias de manejo y conservación adecuadas para este grupo de plantas.

En este estudio se analizan los cambios de las características funcionales de las diez especies de epífitas vasculares más abundantes en el bosque mesófilo de montaña y vegetación secundaria con distinto grado de conservación (bosque conservado, acahual de 20 años y cafetal bajo sombra), así como en cuatro especies que se distribuyen en cada uno de los tres sitios de estudio, en la región central del estado de Veracruz. El objetivo fue analizar la relación entre características funcionales y la variación microclimática determinada por los cambios en la estructura del dosel, ocasionados por las actividades antrópicas. En particular, se analizaron los cambios de la masa seca por unidad de área y variables asociadas a esta característica (área foliar, grosor de la lámina foliar, densidad foliar y contenido de agua). 


\section{Materiales y métodos}

Sitios de estudio. El trabajo fue realizado en tres sitios de estudio ubicados en la región central del estado de Veracruz (Figura 1): (1) un fragmento de bosque mesófilo conservado con exposición suroeste; (2) un acahual de 20 años de edad con exposición sureste, ambos ubicados en el Rancho "Los Capulines" (19³2' 47.1" N y 96 58'30.73" O; altitud entre 1,600 y $1,700 \mathrm{~m})$, municipio de San Andrés Tlalnelhuayocan y; (3) un cafetal bajo sombra ubicado en la localidad de Consolapa, con exposición sureste, municipio de Coatepec (19²9' $10.5^{\prime \prime} \mathrm{N}$ y $96^{\circ} 57^{\prime} 38.9^{\prime \prime}$ O; 1,500 m s.n.m.). El bosque conservado presenta una clara estratificación vertical con un dosel cerrado y una altura entre 20 y $25 \mathrm{~m}$. Los árboles dominantes de este sitio son Quercus xalapensis Bonpl., Q. laurina Bonpl. y Liquidambar styraciflua L., el estrato arbustivo está dominado por especies de la familia Compositae, entre las que sobresalen Roldana sartorii (Sch.Bip. ex Hemls.) H.Rob. \& Bretell y Senecio hartwegii Benth. En este sitio se han registrado 89 especies de epífitas vasculares, las familias con mayor número de taxa son Polypodiaceae (35), Orchidaceae (24), Bromeliaceae (12) y Piperaceae (10; Gómez-Díaz, 2010).

La zona de acahual presenta un dosel más abierto con una altura de $15 \mathrm{~m}$, donde dominan Liquidambar styraciflua y Hedyosmum mexicanum C. Corden, mientras que en el estrato arbustivo, Brunellia mexicana Standl. y Roldana sartorii son las especies más notables. En este sitio se regis- traron 40 especies de epífitas, donde dominan las familias Orchidaceae (17) y Bromeliaceae (10), así como el grupo de Pteridophyta (13; Gómez-Díaz, 2010). El sitio del cafetal bajo sombra tiene una altura del dosel de entre 12 y $15 \mathrm{~m}$, las especies arbóreas dominantes son Persea americana Mill., P. schiedeana Nees, Trema micrantha (L.) Blume, Erythrina america Mill., Xanthoxylon sp. y, existe una gran abundancia de Citrus sinensis (L.) Osbeck en el estrato medio. El estrato arbustivo está integrado casi exclusivamente por individuos de Coffea arabica L. (variedad robusta). En este sitio se han registrado 50 especies de epífitas, en donde las familias mejor representadas son Bromeliaceae (13) y Orchidaceae (11), así como el grupo de Pteridophyta (9; T.M. Susan-Tepetlan, datos no publicados).

Los tres sitios de estudio muestran cambios en la estructura del dosel que están relacionados con la intensidad del manejo o el grado de la conservación de cada sitio. Estos cambios pueden provocar variaciones microclimáticas significativas en el dosel, que afectan la distribución y abundancia de las especies epífitas. El bosque conservado muestra el dosel más cerrado de los tres sitios, seguido por el acahual y el cafetal. En esta región, la apertura del dosel en los cafetales es modificada para garantizar el desarrollo adecuado del café (Cruz et al., 2010). Este cambio en la estructura del dosel se realiza por medio de la reducción de la densidad especies arbóreas, por sustitución de las especies primarias por especies útiles y de rápido crecimiento (p. ej. Citrus sinensis, Trema micrantha), y mediante podas. En el

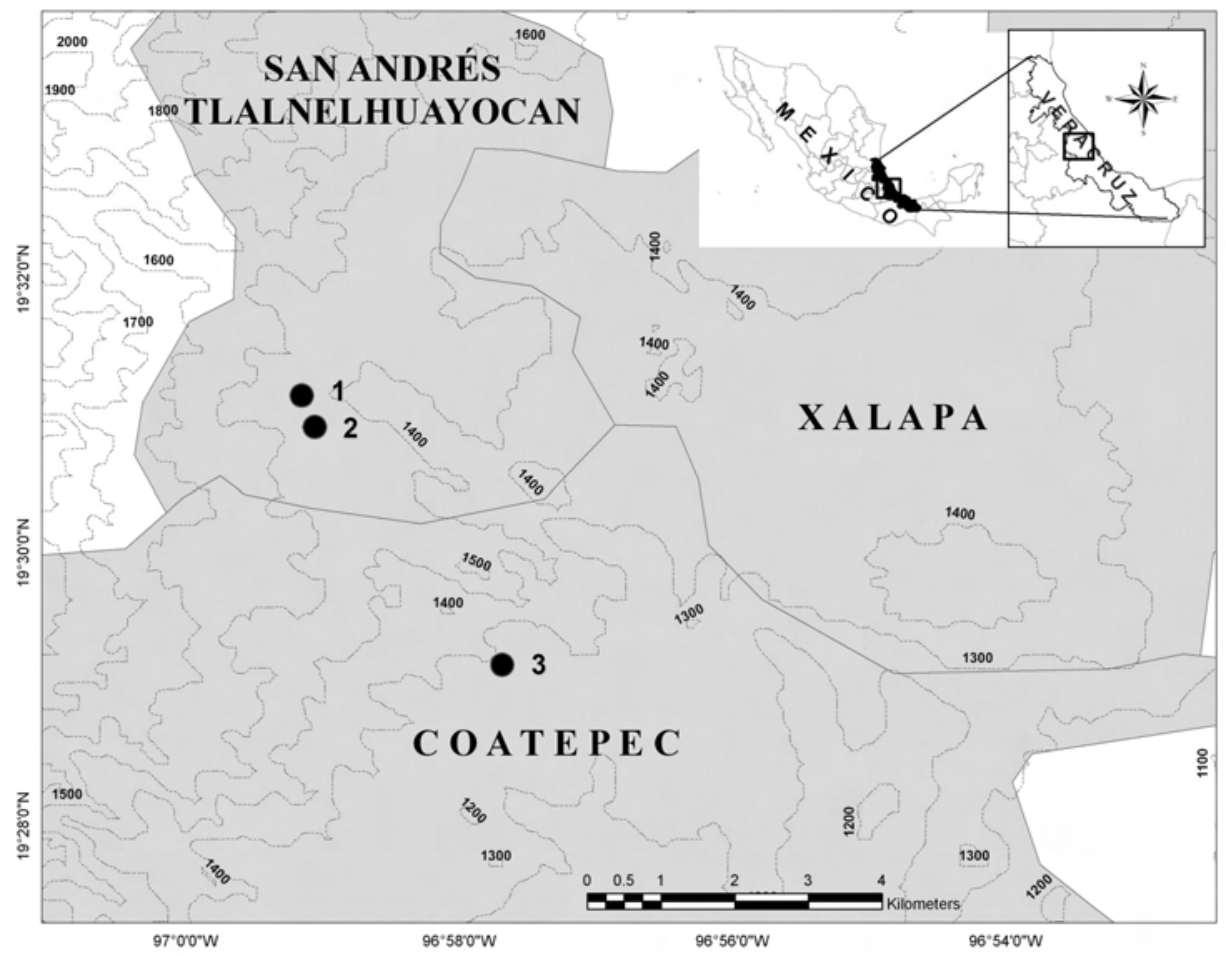

Figura 1. Ubicación de los tres sitios de estudio. Bosque conservado (1), acahual (2) y cafetal bajo sombra (3) en la región central del estado de Veracruz, México. 
caso del acahual, las diferencias de la estructura del dosel respecto al bosque pueden deberse a que la especie arbórea dominante (Liquidambar styraciflua) es secundaria, su arquitectura de copa es menos compleja; es decir, tiene ramas plagiotrópicas con un nivel bajo de ramificación, además de que es caducifolia y se presenta sin hojas durante los meses de noviembre-marzo (Sosa, 1981).

Variables microclimáticas. La temperatura y humedad relativa fueron medidas con dos sensores digitales (Data Logger HOBO U12-013, E.U.A.) por sitio, durante tres meses (mayo-agosto 2012). En caso del cafetal, solo se consideraron los datos de un sensor para la temperatura, ya que los datos del segundo fueron erróneos. Este periodo incluyó el último mes de secas y dos meses de la época de lluvias. Los sensores fueron colocados a $5 \mathrm{~m}$ de altura; es decir, entre la parte superior del tronco y las principales ramificaciones de las copas de los árboles seleccionados para el muestreo de epífitas. Para la descripción del ambiente lumínico de las zonas de colecta, se estimó la apertura del dosel y la transmitancia total de radiación fotosintéticamente activa del dosel (RFA), a través de fotografías hemisféricas (Cámara digital, Colpis A Nikon con un lente de ojo de pescado) y el programa Gap Light Analyzer Watch 2.0 (Frazer et al., 1999). En cada sitio se tomaron diez fotografías en los árboles de colecta a una altura de $5 \mathrm{~m}$. En estos puntos, además se realizaron medidas instantáneas de RFA con un medidor de densidad fotónica (LI-190, LI-COR, Lincoln, Nebraska, E.U.A.). Estas medidas fueron realizadas en dos días (nublados y soleados) entre las 12:00 y 13:00, del mes de septiembre del 2012.

Selección de especies y determinación de características funcionales. En cada sitio, previamente se realizaron muestreos de epífitas del sotobosque en ocho cuadros de $20 \times$ $20 \mathrm{~m}$, así como en cuatro árboles del dosel, siguiendo el método de Gradstein et al. (2003), en los que se determinó la riqueza, composición y abundancia relativa de las epífitas (Gómez-Díaz, 2010; T.M. Susan-Tepetlan, datos no publicados). Con base en estos resultados, se eligieron las diez especies cuya frecuencia en las unidades de muestreo fuera superior al $60 \%$ y que presentaran una abundancia relativa alta en cada sitio, por lo cual el número total especies para los tres sitios de estudio fue de 26 (Apéndice 1). Dentro de este grupo de especies se registraron cuatro (los helechos Phlebodium areolatum (L.) J.Sm., Pleopeltis crassinervata Hum. \& Bonpl. ex Willd. y Polypodium furfuraceum Schltdl. \& Cham., así como la bromelia Catopsis sessiliflora (Ruiz $\&$ Pav.) Mez), que fueron abundantes en los tres sitios. Estas especies fueron consideradas para evaluar sus cambios a nivel intraespecífico en una segunda fase del estudio. En cada sitio, dentro de los cuadros que se utilizaron para los muestreos de epífitas, se seleccionaron árboles con tallas entre los 8 y 10 m que estuvieron ubicados en el estrato me- dio y alto del dosel de cada comunidad. En estos árboles se colectaron cinco individuos sanos, completos y maduros de cada especie de estudio (Pérez-Harguindeguy et al., 2013). Los individuos seleccionados estuvieron ubicados dentro de las zonas verticales 2 y 3, según Johansson (1974), en una altura aproximada de 4 y $6 \mathrm{~m}$. Para cada individuo se colectaron cinco hojas maduras (25 hojas por especie; PérezHarguindeguy et al., 2013), las cuales fueron colocadas en bolsas plásticas para su posterior análisis en el laboratorio de Ecología Fisiológica del Instituto de Ecología, UNAM.

Para determinar el área foliar, en 25 hojas de cada especie se midió su área, excluyendo los pecíolos, con un medidor de área foliar portátil (LICOR 3100C), mientras que en caso de hojas compuestas se consideró al foliolo como la unidad funcional. En el caso de Phlebodium aerolatum, que presenta una lámina continua sin una separación de foliolos del raquis, se consideró el área de todos los lóbulos y las partes foliares del raquis de cada fronda. Posteriormente, a la evaluación del área foliar se midió el peso fresco y se tomó el grosor de la lámina foliar con un micrómetro (SMT212, con una precisión de $0.002 \mathrm{~mm}$ ). Las hojas fueron secadas en un horno a $70{ }^{\circ} \mathrm{C}$ durante $72 \mathrm{~h}$, y se determinó la masa seca por unidad de área, a partir de la cual se calculó la densidad foliar (Poorter et al., 2009). El contenido de agua se calculó tomado como base la masa seca foliar ([(peso fresco - peso seco) (peso seco $\left.\left.)^{-1}\right] \times 100\right)$, expresado en términos porcentuales (Colombo et al., 2008).

Análisis estadísticos. Los promedios de las variables microclimáticas y funcionales fueron comparados entre sitios, mediante un análisis de varianza de una vía (ANDEVA). Este análisis también se utilizó para comparar las características funcionales, a nivel intraespecífico, en las cuatro especies que se presentaron en los tres sitios de estudio. Para el caso de las comparaciones de las características funcionales entre sitios, se consideraron los valores promedio de cada especie como unidades muestrales; mientras que, en las intraespecíficas, fueron los promedios de los individuos de cada especie. Antes de realizar el ANDEVA se comprobó la homogeneidad de varianzas por medio de la prueba de Barlett (Zar, 1998). En los casos en donde se registraron diferencias significativas entre promedios, se realizó una prueba pareada de Tukey (Zar, 1998). Todos los análisis fueron realizados con el programa JMP (8.02, SAS Institute).

\section{Resultados}

Variables microclimáticas. Las variables microclimáticas analizadas mostraron cambios que están asociados con la estructura del dosel de los sitios de estudio. La apertura del dosel fue significativamente más alta en el acahual y el cafetal, en comparación con la del bosque (Cuadros 1, 2). Esto determinó que la transmitancia total de radiación fotosintéticamente activa del dosel fuera, de manera significativa, más 
Cuadro 1. Valores promedio ( $\pm \mathrm{EE})$ de apertura del dosel (AD), transmitancia total de radiación fotosintéticamente activa (TT), radiación fotosintéticamente activa (RFA), temperatura (T) y humedad relativa (HR), para los tres sitios de estudio, en la región central de Veracruz, México. Las letras diferentes indican diferencias significativas entre sitios $(P<0.05)$.

\begin{tabular}{llllll}
\hline Sitio & $\begin{array}{l}\mathrm{AD} \\
(\%)\end{array}$ & $\begin{array}{l}\mathrm{TT} \\
\left(\mathrm{mol} \mathrm{m} \mathrm{m}^{-2} \mathrm{~d}^{-1}\right)\end{array}$ & $\begin{array}{l}\text { RFA } \\
\left(\mu \mathrm{mol} \mathrm{m} \mathrm{m}^{-2} \mathrm{~s}^{-1}\right)\end{array}$ & $\begin{array}{l}\mathrm{T} \\
\left({ }^{\circ} \mathrm{C}\right)\end{array}$ & $\begin{array}{l}\mathrm{HR} \\
(\%)\end{array}$ \\
\hline Bosque & 7.91 & 4.44 & 18.2 & 17.3 & 83.3 \\
conservado & $(0.53)^{\mathrm{b}}$ & $(0.43)^{\mathrm{b}}$ & $(3.2)^{\mathrm{b}}$ & $(0.1)^{\mathrm{a}}$ & $(0.8)^{\mathrm{a}}$ \\
Acahual & 12.01 & 8.27 & 31.8 & 17.4 & 63.3 \\
& $(0.51)^{\mathrm{a}}$ & $(0.29)^{\mathrm{a}}$ & $(2.5)^{\mathrm{b}}$ & $(0.1)^{\mathrm{a}}$ & $(0.8)^{\mathrm{b}}$ \\
Cafetal & 10.95 & 8.58 & 57.1 & 19.7 & 46.8 \\
& $(0.37)^{\mathrm{a}}$ & $(0.40)^{\mathrm{a}}$ & $(7.6)^{\mathrm{a}}$ & $(0.1)^{\mathrm{a}}$ & $(1.2)^{\mathrm{c}}$ \\
\hline
\end{tabular}

alta en ambos sitios (Cuadro 2). En las medidas instantáneas de radiación fotosintéticamente activa, el acahual presentó el promedio más alto (Cuadros 1, 2), este valor fue 1.7 y 3.1 veces más alto en comparación con los valores del cafetal y del bosque, respectivamente.

La temperatura media más alta se registró en el cafetal, dos grados por encima de los valores promedio del bosque conservado y acahual, aunque no se registraron diferencias significativas entre sitios (Cuadros 1,2). De manera contraria, la humedad relativa decreció en función del grado de apertura del dosel; es decir, el promedio más alto fue registrado en el bosque, este valor fue 1.7 veces más alto que el registrado en el cafetal (Cuadros 1,2).

Características funcionales. Los valores promedio de las características funcionales de cada especie se presentan en el Apéndice 1. Los valores promedio del área foliar de las epífitas en los tres sitios de estudio variaron entre $53.4 \mathrm{y}$ $70.1 \mathrm{~cm}^{2}$ (Figura 2A), las especies del bosque conservado presentaron el valor más alto, aunque no se registraron diferencias significativas entre sitios (Cuadro 2). Para el grosor de la lámina foliar, el valor promedio significativamente más alto fue registrado para las especies del cafetal $(0.441 \mathrm{~mm})$, seguido por los valores del acahual $(0.372 \mathrm{~mm})$ y del bosque $(0.299 \mathrm{~mm})$, estos últimos no fueron significativamente distintos entre sí (Figura 2B, Cuadro 2).

El valor promedio significativamente más alto para la masa seca por unidad de área fue registrado en las especies del cafetal $\left(102.7 \mathrm{~g} \mathrm{~cm}^{-2}\right)$ seguido por los valores del acahual $\left(67.5 \mathrm{~g} \mathrm{~cm}^{-2}\right)$ y del bosque (54.4 $\mathrm{g} \mathrm{cm}^{-2}$; Figura 2C). Este comportamiento está relacionado directamente con la densidad foliar, cuyos valores promedio mostraron el mismo comportamiento (Figura 2D). Los valores promedio significativamente más altos de densidad foliar se registraron en el cafetal $\left(0.219 \mathrm{~g} \mathrm{~cm}^{-3}\right)$ y el valor más bajo en el bosque $\left(0.116 \mathrm{~g} \mathrm{~cm}^{-3}\right)$.

Por último, en el contenido de agua se registraron diferencias significativas entre sitios (Cuadro 2; Figura 2E). Las
Cuadro 2. Resultados de los análisis de varianza para los factores microclimáticos y características funcionales. Apertura del dosel (AD), transmitancia total de radiación fotosintéticamente activa (TT), radiación fotosintéticamente activa (RFA), temperatura ( $\mathrm{T}$ ), humedad relativa $(\mathrm{HR})$, área foliar (AF), masa seca por unidad de área (MS), grosor de la lámina foliar (GL), densidad foliar (DF) y contenido de agua (CA). ${ }^{*}=P<0.05,{ }^{* *}=P<0.01,{ }^{* * *}=P<0.001$.

\begin{tabular}{|c|c|c|}
\hline Variables & $F$ & $P$ \\
\hline \multicolumn{3}{|c|}{ Factores microclimáticos } \\
\hline $\mathrm{AD}$ & 19.94 & $* * *$ \\
\hline TT & 35.55 & $* * *$ \\
\hline RFA & 14.06 & $* * *$ \\
\hline $\mathrm{T}$ & 0.005 & n.s. \\
\hline HR & 327 & $* * *$ \\
\hline \multicolumn{3}{|c|}{ Valores de las diez especies más importantes } \\
\hline AF & 0.27 & n.s. \\
\hline MS & 38.3 & $* * *$ \\
\hline GL & 21.46 & $* * *$ \\
\hline DF & 38.27 & $* * *$ \\
\hline $\mathrm{CA}$ & 5.0 & $* * *$ \\
\hline \multicolumn{3}{|c|}{ Comparaciones intraespecíficas } \\
\hline \multicolumn{3}{|c|}{ Catopsis sessiliflora } \\
\hline $\mathrm{AF}$ & 13.22 & $* * *$ \\
\hline MS & 3.66 & * \\
\hline GL & 1.5 & n.s. \\
\hline DF & 3.66 & * \\
\hline CA & 3.96 & * \\
\hline \multicolumn{3}{|c|}{ Phlebodium aerolatum } \\
\hline AF & 6.4 & $* *$ \\
\hline MS & 0.64 & n.s. \\
\hline GL & 9.007 & $* * *$ \\
\hline DF & 0.62 & n.s. \\
\hline $\mathrm{CA}$ & 0.70 & n.s. \\
\hline \multicolumn{3}{|c|}{ Pleopeltis crassinervata } \\
\hline $\mathrm{AF}$ & 0.18 & n.s. \\
\hline MS & 7.31 & $* *$ \\
\hline GL & 0.196 & n.s. \\
\hline DF & 7.35 & $* *$ \\
\hline $\mathrm{CA}$ & 3.51 & $*$ \\
\hline \multicolumn{3}{|c|}{ Polypodium furfuraceum } \\
\hline $\mathrm{AF}$ & 8.42 & $* * *$ \\
\hline MS & 5.01 & $*$ \\
\hline GL & 28.43 & $* * *$ \\
\hline DF & 50.10 & $* *$ \\
\hline $\mathrm{CA}$ & 5.21 & * \\
\hline
\end{tabular}

especies del bosque presentaron los porcentajes promedio significativamente más altos $(760.2 \%)$, seguidas por las de acahual $(558.9 \%)$, y el valor más bajo se presentó en el cafetal $(375.3 \%)$.

En el caso de las comparaciones intraespecíficas, en las cuatro especies, sólo la masa seca por unidad de área y la 

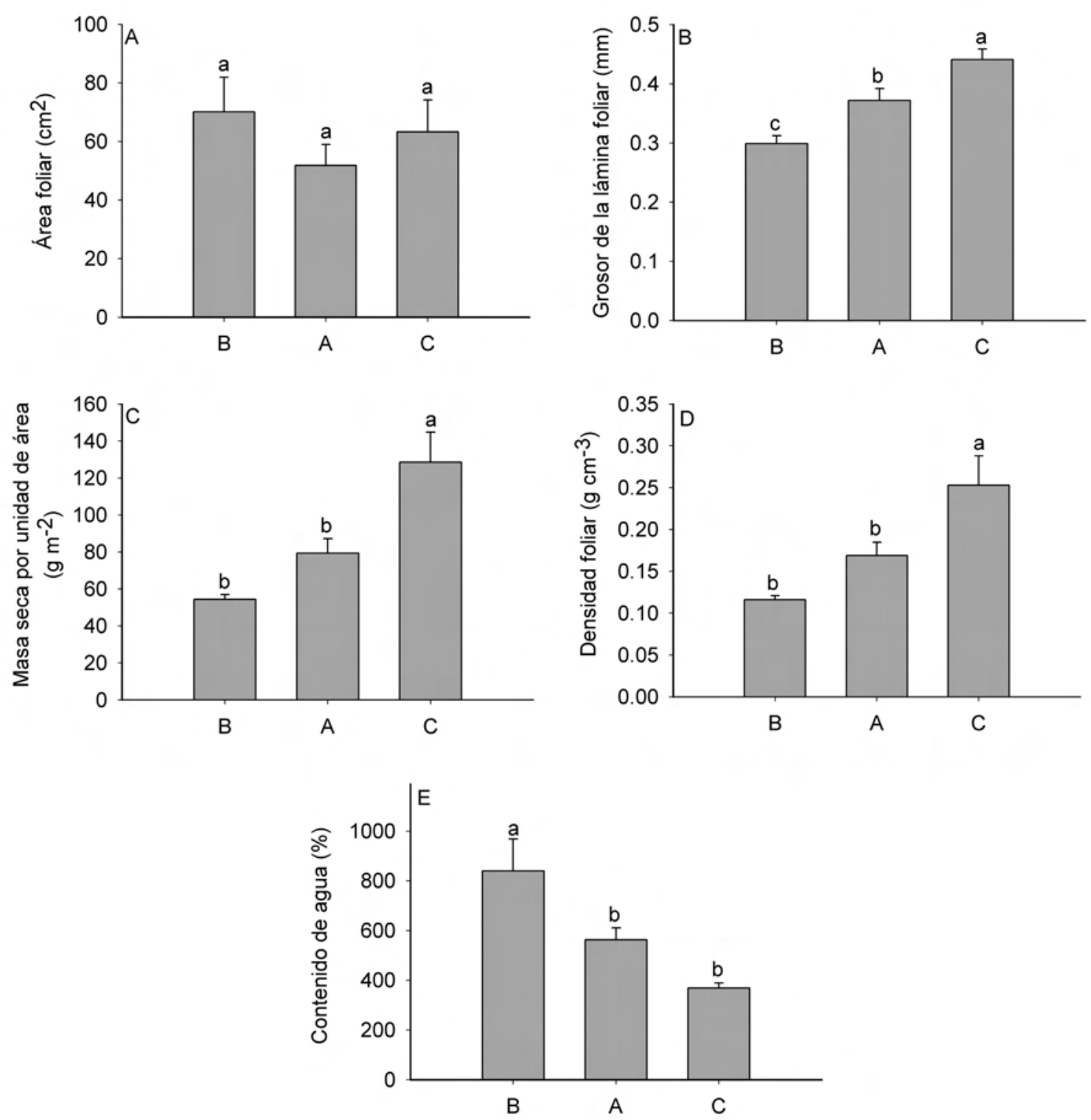

Figura 2. Valores promedio ( \pm E.E.) de las características funcionales evaluadas en las diez especies de epífitas más abundantes en el bosque conservado (B), acahual (A) y cafetal (C). Las letras diferentes sobre las barras indican diferencias significativas entre sitios $(P<$ $0.05)$.

densidad foliar siguieron el mismo patrón observado en las diez especies más abundantes por sitio (Figura 3). Los valores de ambas características se incrementaron significativamente conforme se incrementó el grado de perturbación (Figura 3C, D; Cuadro 2). En el caso del grosor de la lámina foliar, este patrón se registró para los helechos Phlebodium areolatum y Polypodium furfuraceum (Figura 3B, Cuadro 2), mientras que en el área foliar solo para la bromelia $\mathrm{Ca}$ topsis sessiliflora (Figura 3A).

\section{Discusión}

Los cambios ambientales ocasionados por la eliminación o modificación de la estructura del bosque natural causan cambios en la composición, abundancia relativa, distribución espacial y respuestas funcionales de las epífitas (Andrade y Nobel, 1997, Krömer y Gradstein, 2003; Werner et al., 2005; Wolf, 2005; Hietz et al., 2006; Flores-Palacios y García-Franco, 2008; Larrea y Werner, 2010). Los resultados mostraron que el grado de conservación de las comunidades arbóreas analizadas afecta la estructura del dosel, lo que modifica las condiciones microclimáticas y las respuestas funcionales de las epífitas más abundantes de cada sitio.

La estructura del dosel determina el ambiente físico en el que se desarrollan las epífitas, debido a que influye directamente en la temperatura, concentración de vapor y el régimen lumínico (Benzing, 1995; Köster et al., 2011). Estas variables afectan procesos fisiológicos y ecológicos como la 

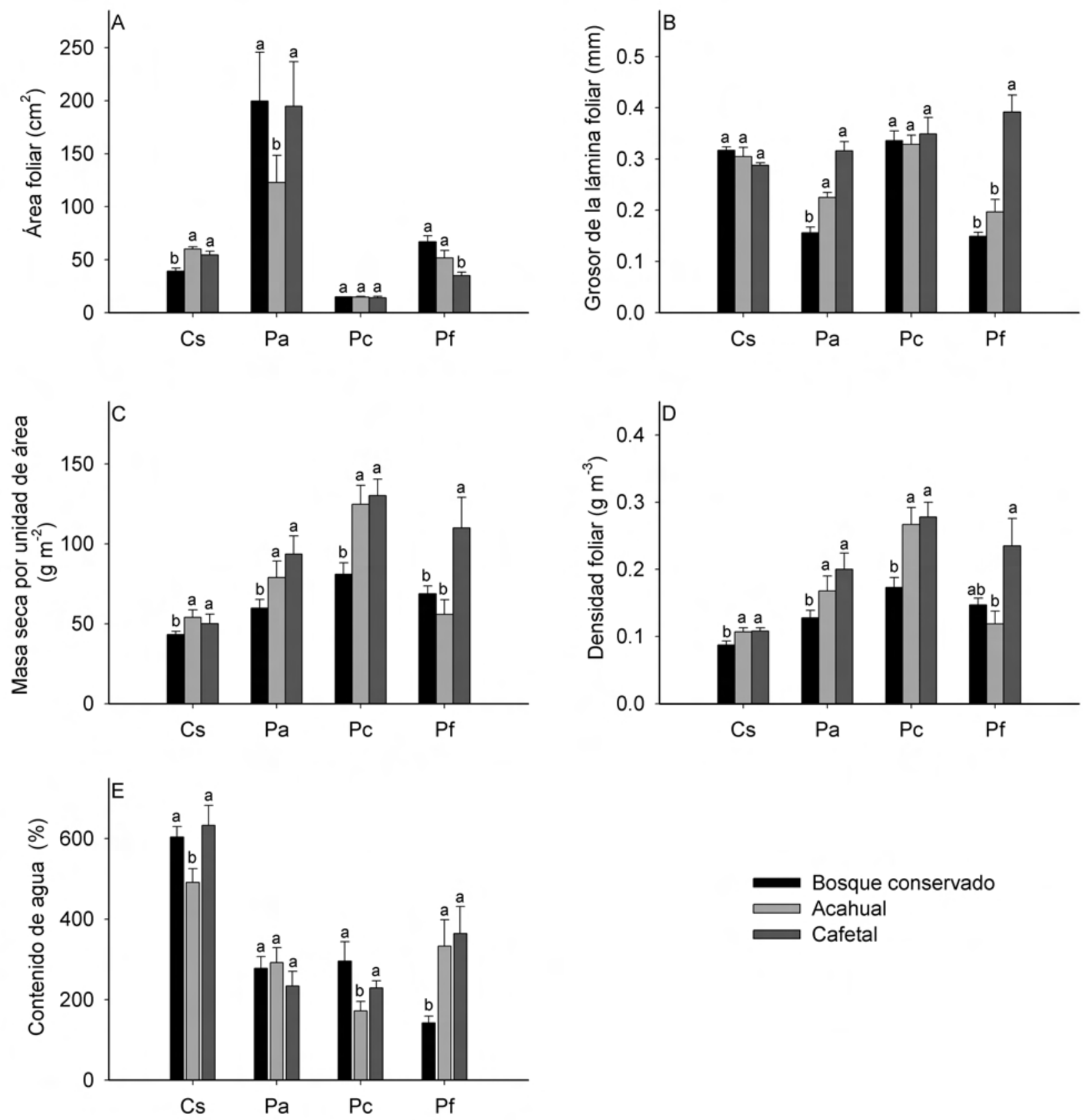

Figura 3. Valores promedio ( \pm E.E.) de las características funcionales evaluadas en Catopsis sessiliflora (Ca), Phlebodium areolatum (Pa), Pleopeltis crassinervata $(\mathrm{Pc})$ y Polypodium furfuraceum $(\mathrm{Pf})$. Las letras diferentes sobre las barras indican diferencias significativas entre sitios $(P<0.05)$.

fotosíntesis, la transpiración, la elongación celular, la fotomorfogénesis y la infestación por patógenos (Campbell y Norman, 1989; Madigosky, 2004; Funk y Lerdau, 2004). En este estudio, en el estrato medio del dosel, en el cafetal es probable que se presenten condiciones de estrés hídrico más frecuentes, en comparación con el acahual y el bosque. Esto se debe a que presentó la apertura de dosel más alta, lo cual determinó que presentará los valores de transmitancia y de radiación fotosintéticamente activa $50 \%$ más altos que los valores promedio del bosque conservado. De manera contraria, la humedad relativa decreció $56 \%$ en cafetal, en comparación con el promedio del bosque. Además, la temperatura del cafetal fue más alta que en el acahual y bosque, influyendo directamente en la reducción de la humedad relativa, debido a que en el dosel de los bosques tropicales la humedad atmosférica está relacionada de manera inversa con la temperatura (Madigosky, 2004).

La escasez de agua en el dosel es una de las restricciones más importantes para la distribución vertical de las epífitas (Andrade y Nobel, 1997; Zotz y Hietz, 2001). Los resulta- 
dos de este trabajo indican que las diferencias registradas en las respuestas funcionales entre sitios están asociadas a las condiciones de estrés hídrico y de alta radiación. Los valores en el grosor de la lámina foliar, masa seca por unidad de área y densidad foliar son más altos en el cafetal, en donde las condiciones de estrés hídrico pueden ser más altas. Esto coincide con los resultados de Hietz y Briones (1998), quienes registraron que el grosor de la lámina foliar de varios helechos epífitos se incrementa cuando están más expuestas en el dosel. El incremento de la masa seca por unidad de área debido a condiciones de estrés hídrico y alta radiación, ha sido explicado por la reducción de la elongación celular y el incremento del grosor de las paredes celulares (Niinemets et al., 1998; Niinemets et al., 2004). En hojas con altos valores de la masa seca por unidad de área, las células del mesófilo son más pequeñas y se compactan, reduciendo los espacios intercelulares y provocando un incremento en la densidad y una disminución del volumen foliar (Poorter et al., 2009). En las epífitas, algunas de las respuestas anatómicas ante el estrés hídrico son la presencia de paredes y cutículas gruesas en las células de la epidermis, así como una clara diferenciación de la hipodermis y del clorénquima, los cuales generalmente son multiestratificados (Benzing, 1990; Zotz y Andrade, 2002). En conjunto, estas repuestas funcionales pueden favorecer la conservación de agua bajo condiciones de estrés hídrico (Poorter et al., 2009), y pueden explicar las modificaciones en la masa seca por unidad de área y la densidad foliar, registradas en las especies estudiadas, debido a que estas variables se incrementaron en la medida en que se redujo la humedad relativa, se incrementó la temperatura y la radiación fotosintéticamente activa de los sitios. Aunque en el caso de los helechos (excluyendo a Phlebodium areolatum, que es una especie caducifolia), es probable que los cambios de la masa seca por unidad de área y densidad foliar estén asociados a procesos de poiquilohidría (desecación-hidratación; Hietz y Briones, 2004).

Los patrones de cambio de la masa seca por unidad de área y densidad foliar, registrados en las especies más abundantes, fueron consistentes en las cuatro especies que se distribuyen en los tres sitios de estudio. En todos los casos, ambas variables se incrementaron en el sitio del cafetal, que cuenta con menor humedad relativa, pero temperaturas y radiación más altas. Esto sugiere que en las cuatro especies presentes en todos ambientes, las modificaciones en la masa seca por unidad de área y densidad foliar son respuestas que ayudan a enfrentar condiciones de estrés hídrico y alta radiación. La plasticidad de estas variables sería un atributo que permite que estas especies se distribuyan en sitios con condiciones microclimáticas heterogéneas. La mayor plasticidad fenotípica ante la variación ambiental es una característica común en especies de amplia distribución (Sultan, 2001; Valladares et al., 2007).

En este estudio se pudo comprobar que la masa seca por unidad de área y la densidad foliar pueden ayudar entender las respuestas funcionales de las especies de epífitas a los cambios microclimáticos causados por la modificación de la estructura del dosel, debidas al grado de perturbación del bosque mesófilo. Sin embargo, es necesario realizar estudios experimentales más detallados que nos permitan explicar con mayor precisión como influyen los factores ambientales en los cambios de la masa seca por unidad de área y de la densidad foliar de las epífitas. Así como evaluar la importancia de estas variables ante la variación ambiental, su utilidad como índices del estado de conservación de las comunidades y su repercusión en los procesos ecológicos del dosel de los bosques húmedos tropicales.

\section{Agradecimientos}

Queremos agradecer a R. Ortega-Ortíz, J.L. Salazar y A. Orozco-Segovia por su apoyo en el trabajo de campo y por las facilidades otorgadas para el uso de material técnico. A T. Toledo-Aceves, B. Ruiz-Guerra, a los revisores anónimos por las observaciones y valiosos comentarios al manuscrito, y a S. Armenta-Montero por la elaboración del mapa. La investigación fue financiada con fondos de PROMEP (PROMEP/103.5/07/2753) otorgados a Thorsten Krömer, así como una beca del CONACyT (No. 235995) otorgada a T.M. Susan-Tepetlan.

\section{Literatura citada}

Andrade J.L. y Nobel P.S. 1997. Microhabitat and water relation of epiphytic cacti and ferns in a lowland neotropical forest. Biotropica 29:261-270.

Barthlott W., Schmit-Neuerburg V., Nieder J. y Engwald S. 2001. Diversity and abundance of vascular epiphytes: A comparison of secondary vegetation and primary montane rain forest in the Venezuelan Andes. Plant Ecology 152:145-156.

Benzing D.H. 1990. Vascular Epiphytes. General Biology and Related Biota. Cambridge University Press, Cambridge.

Benzing D.H. 1995. The physical mosaic and plant variety in forest canopies. Selbyana 16:159-168.

Benzing D.H. 1998. Vulnerabilities of tropical forests to climate change: the significance of resident epiphytes. Climate Change 39:519-540.

Benzing D.H. 2000. Bromeliaceae: Profile of an Adaptative Radiation. Cambridge University Press, Cambridge.

Campbell G.S. y Norman J.M. 1989. The description and measurement of plant canopy structure. En: Russel G., Marshall B. y Jarvis P.G. Eds. Plant Canopies: Their Growth, Form and Function, pp. 1-20, Cambrige University Press, Cambrige.

Cardelús C.L. y Chazdon R.L. 2005. Inner-crown microenvironments of two emergent tree species in a lowland wet forest. Biotropica 37:238-244.

Colombo R., Meroni M., Marchesi A., Busetto L., Rossini M., Giardino C. y Panigada C. 2008. Estimation of leaf and canopy water content in poplar plantations by means of hyperspectral indices and inverse modeling. Remote Sensing of Environment 112:1820-1834.

CONABIO. Comisión Nacional para el Conocimiento y Uso de la 
Biodiversidad. 2010. El Bosque Mesófilo de Montaña en México: Amenazas y Oportunidades para su Conservación y Manejo Sostenible. Comisión Nacional para el Conocimiento y Uso de la Biodiversidad, México D.F.

Coxson D.S. y Nadkarni N.M. 1995. Ecological roles of epiphytes in nutrient cycles of forest ecosystems. En: Lowman M.D. y Nadkarni N.M. Eds. Forest Canopies, pp. 495-543, Academic Press, San Diego.

Cruz A.A., Escobar S.F., Gerez F.P., Muñiz C.M.A., Ramírez R.F. y Williams L.G. 2010. Centro de Veracruz. En: Comisión Nacional para el Conocimiento y Uso de la Biodiversidad Ed. El Bosque Mesófilo de Montaña en México: Amenazas y Oportunidades para su Conservación y Manejo Sostenible, pp. 80-87, Comisión Nacional para el Conocimiento y Uso de la Biodiversidad, México D.F.

Díaz S., Fargione J., Chapin F.S.III y Tilman D. 2006. Biodiversity loss threatens human well-being. PLOS Biology 4(8):e277.

Flores-Palacios A. y García-Franco J.G. 2008. Habitat isolation changes the beta diversity of the vascular epiphyte community in lower montane forest, Veracruz, Mexico. Biodiversity and Conservation 17:191-207.

Flores-Palacios A. y Valencia-Díaz S. 2007. Local illegal trade reveals unknown diversity and involves a high species richness of wild vascular epiphytes. Biological Conservation 136:372387.

Flores-Palacios A., García-Franco J.G., Valencia-Díaz S., SolísMontero L. y Cruz-Angón A. 2011. Diversidad y conservación de plantas epífitas vasculares en el centro del Estado. En: Comisión Nacional para el Conocimiento y Uso de la Biodiversidad, Ed. La Biodiversidad en Veracruz: Estudio de Estado, Vol. I, pp. 493-501, Comisión Nacional para el Conocimiento y Uso de la Biodiversidad, Gobierno del Estado de Veracruz, Universidad Veracruzana, Instituto de Ecología, A.C., México D.F.

Foster P. 2001. The potential negative impacts of global climate change on tropical montane cloud forests. Earth-Science Reviews 55:73-106.

Frazer G.W., Canham C.D. y Lertzman K.P. 1999. Gap Light Analyser (GLA), Version 2.0: Imagingsoftware to extract canopy structure and gap light transmission indices from truecolour fisheye photographs User's manual and program documentation. Simon Frazer University, Institute of Ecosystems Studies, Millbrook.

Funk J.L. y Lerdau M.T. 2004. Photosynthesis in forest canopies. En: Lowman M.D. y Rinker H.B. Eds. Forest Canopies, $2^{\mathrm{a}}$ ed., pp. 335-358, Elsevier Academic Press, San Diego.

Garnier E., Cortez J., Billès G., Navas M.L., C. Roumet, Debussche M., Laurent G., Blanchard A., Aubry D., Bellmann A., Neill C. y Toussaint J.P. 2004. Plant functional markers capture ecosystem properties during secondary succession. Ecology 85:2630-2637.

Gómez-Díaz J.A. 2010. Comparación florística de epífitas vasculares entre un bosque mesófilo de montaña y un acahual en el Municipio de Tlalnelhuayocan, Ver. Tesis de Licenciatura, Facultad de Ciencias Biológicas y Agropecuarias, Zona Xalapa, Universidad Veracruzana. 117 pp.

Gradstein S.R. 2008. Epiphytes of tropical montane forests - impact of deforestation and climate change. En: Gradstein S.R., Homeier J. y Gansert D. Eds. The Tropical Montane Forest: Patterns and Processes in a Biodiversity Hotspot, pp. 51-65, Universitätsverlag Göttingen, Göttingen.
Gradstein S.R., Nadkarni N.M., Krömer T., Holz I. y Nöske N 2003. A protocol for rapid and representative sampling of vascular and non-vascular epiphyte diversity in tropical rain forests. Selbyana 24:105-111.

Hietz P. 1998. Diversity and Conservation of Epiphytes in a Changing Environment. Invited lecture presented at the International Conference on Biodiversity and Bioresources: Conservation and Utilization, 23-27 November 1997, Phuket, Thailand. Pure and Applied Chemistry 70:2114-2124.

Hietz P. 2010. Ecology and ecophysiology of epiphytes in tropical montane cloud forests. En: Bruijnzeel L.A., Scatena F.N. y Hamilton L.S. Eds. Tropical Montane Cloud Forests: Science for Conservation and Management, pp. 67-76, Cambridge University Press, Cambridge.

Hietz P. y Briones O. 1998. Corrrelation between water relations and within-canopy distribution of epiphytic ferns in a Mexican cloud forest. Oecologia 114:305-316.

Hietz P. y Briones O. 2004. Adaptaciones y bases fisiológicas de la distribución de los helechos epifitos en un bosque de niebla de México. En: Marino H. Ed. Fisiología Ecológica en Plantas, pp. 121-138, Ediciones Universitarias de Valparaíso, Valparaíso.

Hietz P. y Wanek W. 2003. Size-dependent variation of carbon and nitrogen isotope abundance in epiphytic bromeliads. Plant Biology 5:137-142.

Hietz P., Buchberger G. y Winkler M. 2006. Effect of forest disturbance on abundance and distribution of epiphytic bromeliads and orchids. Ecotropica 12:103-112.

Johansson D. 1974. Ecology of vascular epiphytes in west African rain forest. Acta Phytogeographica Suecica 59:1-29.

Kelly D.L., Tanner E.V.J., Lughadha E.M. y Kapos V. 1994. Floristics and biogeography of a rain forest in the Venezuelan Andes. Journal of Biogeography 21:421-440.

Kessler M. 2001. Maximum plant-community endemism at intermediate intensities of anthropogenic disturbance in Bolivian montane forests. Conservation Biology 15:634-641.

Köster N., Nieder J. y Barthlott W. 2011. Effect of host tree traits on epiphyte diversity in natural and anthropogenic habitats in Ecuador. Biotropica 43:685-694.

Köster N., Friedrich K., Nieder J. y Barthlott W. 2009. Conservation of epiphyte diversity in an Andean landscape transformed by human land use. Conservation Biology 23:911-919.

Köhler L., Tobón C., Frumau K.F.A. y Bruijnzeel L.A. 2007. Biomass and water storage dynamics of epiphytes in old-growth and secondary montane cloud forest stands in Costa Rica. Plant Ecology 193:171-184.

Krömer T. y Gradstein S.R. 2003. Species richness of vascular epiphytes in two primary forests and fallows in the Bolivian Andes. Selbyana 24:190-195.

Krömer T., García-Franco J.G. y Toledo-Aceves T. En prensa. Epífitas vasculares como bioindicadores de la calidad forestal: impacto antrópico sobre su diversidad y composición. En: González-Zuarth C.A., Vallarino-Moncada A. y Rendón von Osten J. Eds. Los Bioindicadores ¿Guardianes de Nuestro Futuro Ecológico?, Universidad Autónoma de Campeche, San Francisco de Campeche.

Krömer, T. Kessler M. y Gradstein S.R. 2007. Vertical stratification of vascular epiphytes in submontane and montane forest of the Bolivian Andes: the importance of the understory. Plant Ecology 189: 261-278.

Krömer T., Kessler M., Gradstein S.R. y Acebey A. 2005. Diversi- 
ty patterns of vascular epiphytes along an elevational gradient in the Andes. Journal of Biogeography 32:1799-1809.

Larrea M.L. y Werner F.A. 2010. Response of vascular epiphyte diversity to different land-use intensities in a neotropical montane wet forest. Forest Ecology and Management 260:1950-1955.

Madigosky S.R. 2004. Tropical microclimatic considerations. En: Lowman M.D. y Rinker H.B. Eds. Forest Canopies, $2^{\mathrm{a}}$ ed., pp. 24-48, Elsevier Academic Press, San Diego.

Martínez-Garza C., Peña V., Ricker M., Campos A. y Howe H.F. 2005. Restoring tropical biodiversity: Leaf traits predict growth and survival of late-successional trees in early-successional environments. Forest Ecology and Management 217:365-379.

MEA. Millennium Ecosystem Assessment. 2005. Ecosystems and Human Well-being: Biodiversity Synthesis. World Resources Institute, Washington, DC.

Nadkarni N.M. y Solano R. 2002. Potential effects of climate change on canopy communities in a tropical cloud forest: An experimental approach. Oecologia 131:580-586.

Niinemets Ü., Kull O. y Tenhunen J.D. 1998. An analysis of light effects on foliar morphology, physiology, and light interception in temperate deciduous woody species of contrasting shade tolerance. Tree Physiology 18:681-696.

Niinemets Ü., Kull O. y Tenhunen J.D. 2004. Within-canopy variation in the rate of development of photosynthetic capacity is proportional to integrated quantum flux density in temperate deciduous trees. Plant, Cell and Environment 27:293-313.

Pérez-Harguindeguy N., Díaz S., Garnier E., Lavorel S., Poorter H., Jaureguiberry P., Bret-Harte M.S., Cornwell W.K., Craine J.M., Gurvich D.E., Urcelay C., Veneklaas E.J., Reich P.B., Poorter L., Wright I.J., Ray P., Enrico L., Pausas J.G., de Vos A.C., Buchmann N., Funes G., Quétier F., Hodgson J.G., Thompson K., Morgan H.D., ter Steege H., van der Heijden M.G.A., Sack L., Blonder B., Poschlod P., Vaieretti M.V., Conti G., Staver A.C., Aquino S. y Cornelissen J.H.C. 2013. New handbook for standardised measurement of plant functional traits worldwide. Australian Journal of Botany 61:167-234.

Pittendrigh C.S. 1948. The bromeliad-Anopheles-malaria complex in Trinidad. I-The bromeliad flora. Evolution 2:58-89.

Poorter H., Niinemets Ü., Poorter L., Wrigth I.J. y Villar R. 2009. Causes and consequences of variation in leaf mass per area (LMA): a meta-analysis. Tansley Review. New Phytologist 182:565-588.

Rzedowski J. 1996. Análisis preliminar de la flora vascular de los bosques mesófilos de montaña de México. Acta Botanica Mexicana 35:25-44.

Sosa V. 1981. Hamamelidaceae. Flora de Veracruz 1:1-6.

Sultan S.E. 2001. Phenotypic plasticity for fitness components in Polygonum species of contrasting ecological breadth. Ecology 82:328-343.

Toledo-Aceves T., García-Franco J.G., Hernández-Rojas A. y MacMillan K. 2012. Recolonization of vascular epiphytes in a shaded coffee agroecosystem. Applied Vegetation Science 15:99-107.

Turner I.M., Tan H.T.W., Wee Y.C., Ibrahim A.B., Chew P.T. y Corlett R.T. 1994. A study of plant species extinction in Singapore: lessons for the conservation of tropical biodiversity. Conservation Biology 8:705-712.

Valladares F., Gianoli E. y Gómez J.M. 2007. Ecological limits to plant phenotypic plasticity. New Phytologist 176:749-763.

Werner F.A., Homeier J. y Gradstein S.R. 2005. Diversity of vascular epiphytes on isolated remnant trees in the montane forest belt of Southern Ecuador. Ecotropica 11:21-40.

Williams-Linera G. 2007. El Bosque de Niebla del Centro de Veracruz: Ecología, Historia y Destino en Tiempos de Fragmentación y Cambio Climático. Comisión Nacional para la Biodiversidad, Instituto de Ecología, A.C., Xalapa.

Wolf J.H.D. 2005. The response of epiphytes to anthropogenic disturbance of pine-oak forests in the highlands of Chiapas, Mexico. Forest Ecology and Management 212:376-393.

Zar J.H. 1998. Biostatiscal Analysis. $4^{\text {a }}$ ed., Prentice Hall, Nueva Jersey.

Zotz G. 2013. The systematic distribution of vascular epiphytes - a critical update. Botanical Journal of the Linnean Society 171:453-481.

Zotz G. y Andrade J. 2002. La ecología y fisiología de las epífitas y hemiepífitas. En: Guariguata M. y Kattan G.H. Eds. Ecología y Conservación de Bosques Neotropicales, pp. 271-296, Libro Universitario Regional del Instituto Tecnológico de Costa Rica, San José.

Zotz G. y Bader M.Y. 2009. Epiphytic plants in a changing worldglobal: Change effects on vascular and non-vascular epiphytes. Progress in Botany 70:147-170.

Zotz G. y Hietz P. 2001. The physiological ecology of vascular epiphytes: current knowledge, open questions. Journal of Experimental Botany 364:2067-2078.

Recibido: 4 de noviembre de 2013

Aceptado: 16 de enero de 2014 
RASGOS FUNCIONALES DE EPÍFITAS VASCULARES

Apéndice 1. Valores promedio ( \pm E.E.) de área foliar (AF), grosor de la lámina foliar (GL), masa seca por unidad de área (MS), densidad foliar (DF) y contenido de agua (CA) para todas las especies en los tres sitios de estudio. Abreviaciones de familias: $\mathrm{Ar}=$ Araceae, $\mathrm{Br}=$ Bromeliaceae, $\mathrm{Or}=$ Orchidaceae, $\mathrm{Pi}=$ Piperaceae, $\mathrm{Po}=$ Polypodiaceae)

\begin{tabular}{|c|c|c|c|c|c|}
\hline Sitios/especies (familias) & $\begin{array}{l}\mathrm{AF} \\
\left(\mathrm{cm}^{2}\right)\end{array}$ & $\begin{array}{l}\mathrm{GL} \\
(\mathrm{mm})\end{array}$ & $\begin{array}{l}\text { MS } \\
\left(\mathrm{g} \mathrm{m}^{-2}\right)\end{array}$ & $\begin{array}{l}\text { DF } \\
\left(\mathrm{g} \mathrm{cm}^{-3}\right)\end{array}$ & $\begin{array}{l}\text { CA } \\
(\%)\end{array}$ \\
\hline \multicolumn{6}{|l|}{ Bosque conservado } \\
\hline Catopsis sessiliflora (Ruiz \& Pav.) Mez (Br) & $39.1(2.9)$ & $0.317(0.007)$ & $43.1(2.1)$ & $0.087(0.006)$ & $604.0(25.5)$ \\
\hline Dichaea glauca (Sw.) Lindl. (Or) & $4.9(0.1)$ & $0.216(0.003)$ & $44.2(1.7)$ & $0.094(0.004)$ & $364.2(17.4)$ \\
\hline Phlebodium aerolatum (L.) J.Sm. (Po) & $199.7(46.2)$ & $0.156(0.011)$ & $59.8(5.3)$ & $0.128(0.011)$ & $278.2(28.7)$ \\
\hline Pleopeltis angusta Hum. \& Bonpl. Ex Willd. (Po) & $27.3(1.9)$ & $0.112(0.007)$ & $76.5(7.3)$ & $0.163(0.015)$ & $194.4(37.9)$ \\
\hline Pleopeltis crassinervata (Fée) T.Moore (Po) & $14.9(0.9)$ & $0.336(0.019)$ & $80.9(7.2)$ & $0.173(0.015)$ & $295.4(48.8)$ \\
\hline Peperomia dendrophila Schltdl. \& Cham. (Pi) & $8.2(1.0)$ & $0.554(0.035)$ & $29.6(3.5)$ & $0.063(0.007)$ & $1491.9(104.9)$ \\
\hline Polypodium furfuraceum Schltdl. \& Cham. (Po) & $66.9(5.6)$ & $0.149(0.008)$ & $68.8(4.8)$ & $0.147(0.010)$ & $152.1(17.1)$ \\
\hline Peperomia quadrifolia (L.) Kunth (Pi) & $0.9(0.04)$ & $0.433(0.019)$ & $37.7(1.4)$ & $0.037(0.003)$ & $1377.4(350.7)$ \\
\hline Tillandsia butzii Mez (Br) & $37.0(0.9)$ & $0.314(0.007)$ & $84.8(5.8)$ & $0.181(0.013)$ & $522.4(85.6)$ \\
\hline Tillandsia multicaulis Steud. (Br) & $129.9(14.0)$ & $0.350(0.006)$ & $57.6(2.9)$ & $0.123(0.006)$ & $434.0(30.5)$ \\
\hline \multicolumn{6}{|l|}{ Acahual } \\
\hline Catopsis sessiliflora (Ruiz \& Pav.) Mez (Br) & $60.0(2.3)$ & $0.305(0.018)$ & $53.9(2.9)$ & $0.107(0.006)$ & $491.5(33.8)$ \\
\hline Catopsis nitida (Hook.) Griseb. (Br) & $25.6(3.2)$ & $0.219(0.016)$ & $103.9(15.0)$ & $0.222(0.032)$ & $276.7(71.6)$ \\
\hline Dichaea glauca (Sw.) Lindl. (Or) & $4.7(0.2)$ & $0.232(0.003)$ & $44.9(3.2)$ & $0.095(0.007)$ & $221.9(36.9)$ \\
\hline Phlebodium aerolatum (L.) J.Sm. (Po) & $122.9(25.7)$ & $0.225(0.010)$ & $78.9(10.4)$ & $0.168(0.022)$ & $292.2(37.3)$ \\
\hline Pleopeltis crassinervata (Fée) T.Moore (Po) & $14.7(0.8)$ & $0.329(0.018)$ & $124.8(11.8)$ & $0.267(0025)$ & $171.9(23.4)$ \\
\hline Peperomia dendrophila Schltdl. \& Cham. (Pi) & $5.9(0.4)$ & $0.519(0.023)$ & $27.6(3.1)$ & $0.058(0.006)$ & $1282.1(150.4)$ \\
\hline Polypodium furfuraceum Schltdl. \& Cham. (Po) & $51.7(7.1)$ & $0.197(0.024)$ & $55.9(9.1)$ & $0.119(0.019)$ & $332.9(65.5)$ \\
\hline Peperomia quadrifolia (L.) Kunth (Pi) & $0.9(0.03)$ & $0.662(0.065)$ & $17.7(3.9)$ & $0.080(0.008)$ & $381.3(92.79)$ \\
\hline Tillandsia butzii Mez (Br) & $19.5(3.5)$ & $0.739(0.065)$ & $106.1(5.9)$ & $0.226(0.012)$ & $737.6(43.6)$ \\
\hline Tillandsia multicaulis Steud. (Br) & $130.9(7.5)$ & $0.307(0.013)$ & $70.8(7.3)$ & $0.151(0.015)$ & $363.1(20.2)$ \\
\hline \multicolumn{6}{|l|}{ Cafetal } \\
\hline Anthurium scandens (Aubl.) Engl. (Ar) & $25.1(1.9)$ & $0.482(0.011)$ & $90.6(5.3)$ & $0.194(0.011)$ & $410.8(13.6)$ \\
\hline Catopsis sessiliflora (Ruiz \& Pav.) Mez (Br) & $54.3(3.6)$ & $0.288(0.005)$ & $50.6(2.7)$ & $0.108(0.005)$ & $633.0(49.2)$ \\
\hline Maxillaria densa Lindl. (Or) & $92.7(8.1)$ & $0.525(0.024)$ & $124.8(3.2)$ & $0.267(0.007)$ & $304.2(10.0)$ \\
\hline Phlebodium aerolatum (L.) J.Sm. (Po) & $194.7(42.1)$ & $0.316(0.018)$ & $93.6(11.3)$ & $0.200(0.024)$ & $234.0(36.2)$ \\
\hline Pleopeltis angusta Hum. \& Bonpl. Ex Willd. (Po) & $17.5(1.6)$ & $0.365(0.24)$ & $137.2(8.8)$ & $0.293(0.018)$ & $178.6(16.4)$ \\
\hline Pleopeltis crassinervata (Fée) T.Moore (Po) & $14.0(1.4)$ & $0.349(0.032)$ & $130.3(10.4)$ & $0.278(0.022)$ & $228.8(18.2)$ \\
\hline Polypodium furfuraceum Schltdl. \& Cham. (Po) & $35.1(3.0)$ & $0.392(0.033)$ & $109.9(19.2)$ & $0.235(0.041)$ & $430.58(87.9)$ \\
\hline Peperomia glabella (Sw.) A.Dietr. (Pi) & $4.9(0.2)$ & $0.878(0.055)$ & $108.3(14.7)$ & $0.231(0.031)$ & $496.7(78.9)$ \\
\hline Prosthechea ochracea (Lindl.) W.E.Higgins (Or) & $16.4(0.6)$ & $0.403(0.003)$ & $63.7(1.6)$ & $0.136(0.003)$ & $379.7(18.7)$ \\
\hline Peperomia tetraphylla Hook. \& Arn. (Pi) & $1.1(0.09)$ & $0.463(0.018)$ & $161.1(18.9)$ & $0.344(0.040)$ & $421.4(78.5)$ \\
\hline
\end{tabular}

